\title{
ACPA-positive and ACPA-negative rheumatoid arthritis differ in their requirements for combination DMARDs and corticosteroids: secondary analysis of a randomized controlled trial
}

\author{
Seth D Seegobin ${ }^{1}$, Margaret HY Ma², Chanaka Dahanayake², Andrew P Cope², David L Scott ${ }^{3}$, \\ Cathryn M Lewis ${ }^{1}$ and lan C Scott ${ }^{1,2^{*}}$
}

\begin{abstract}
Introduction: UK guidelines recommend that all early active rheumatoid arthritis (RA) patients are offered combination disease-modifying antirheumatic drugs (DMARDs) and short-term corticosteroids. Anti-citrullinated protein antibody (ACPA)-positive and ACPA-negative RA may differ in their treatment responses. We used data from a randomized controlled trial - the Combination Anti-Rheumatic Drugs in Early RA (CARDERA) trial - to examine whether responses to intensive combination treatments in early RA differ by ACPA status.
\end{abstract}

Methods: The CARDERA trial randomized 467 early active RA patients to receive: (1) methotrexate, (2) methotrexate/ciclosporin, (3) methotrexate/prednisolone or (4) methotrexate/ciclosporin/prednisolone in a factorial-design. Patients were assessed every six months for two years. In this analysis we evaluated 431 patients with available ACPA status. To minimize multiple testing we used a mixed-effects repeated measures ANOVA model to test for an interaction between ACPA and treatment on mean changes from baseline for each outcome (Larsen, disease activity scores on a 28-joint count (DAS28), Health Assessment Questionnaire (HAQ), EuroQol, SF-36 physical component summary (PCS) and mental component summary (MCS) scores). When a significant interaction was present, mean changes in outcomes were compared by treatment group at each time point using t-tests stratified by ACPA status. Odds ratios (ORs) for the onset of new erosions with treatment were calculated stratified by ACPA.

Results: ACPA status influenced the need for combination treatments to reduce radiological progression. ACPA-positive patients had significant reductions in Larsen score progression with all treatments. ACPA-positive patients receiving triple therapy had the greatest benefits: two-year mean Larsen score increases comprised 3.66 (95\% confidence interval (Cl) 2.27 to 5.05 ) with triple therapy and 9.58 (95\% Cl 6.76 to 12.39 ) with monotherapy; OR for new erosions with triple therapy versus monotherapy was 0.32 ( $95 \% \mathrm{Cl} 0.14$ to $0.72 ; P=0.003$ ). ACPA-negative patients had minimal radiological progression irrespective of treatment. Corticosteroid's impact on improving DAS28/PCS scores was confined to ACPA-positive RA.

Conclusions: ACPA status influences the need for combination DMARDs and high-dose tapering corticosteroids in early RA. In CARDERA, combination therapy was only required to prevent radiological progression in ACPA-positive patients; corticosteroids only provided significant disease activity and physical health improvements in ACPA-positive disease. This suggests ACPA is an important biomarker for guiding treatment decisions in early RA.

Trial registration: Current Controlled Trials ISRCTN32484878

\footnotetext{
* Correspondence: ian.scott@kcl.ac.uk

'Department of Medical and Molecular Genetics, King's College London,

Guy's Hospital, Great Maze Pond, 8th Floor Tower Wing, London SE1 9RT, UK

${ }^{2}$ Academic Department of Rheumatology, Centre for Molecular and Cellular

Biology of Inflammation, 1st Floor, New Hunt's House, Guy's Campus, King's

College London, Great Maze Pond, London SE1 1UL, UK

Full list of author information is available at the end of the article
} 


\section{Introduction}

Rheumatoid arthritis (RA) is a heterogeneous disease spanning several subsets. One crucial subdivision is defined by the presence or absence of anti-citrullinated protein antibodies (ACPA), termed ACPA-positive and ACPA-negative RA, respectively [1]. ACPA-positive RA has a worse prognosis with higher rates of erosive damage [2]. It also has different risk factors than ACPA-negative RA with most genetic associations $[3,4]$ and environmental risks, such as smoking [5] and alcohol abstinence [6], predominantly linked to ACPA-positive disease. These disparities suggest that RA ACPA subsets might respond differently to treatment [7].

Current RA management focuses on early intensive therapies, often using combinations of disease-modifying antirheumatic drugs (DMARDs) and glucocorticoids with rapid escalation to biologics in refractory cases. Guideline recommendations for the treatment of early RA differ across countries. UK guidelines from the National Institute for Health and Care Excellence (NICE) advocate that all individuals with active RA are offered combination DMARDs with short-term glucocorticoids [8]. American College of Rheumatology (ACR) guidelines suggest reserving combination DMARDs for patients with markers of severe disease, such as ACPA positivity [9]. The European League Against Rheumatism (EULAR) guidelines also suggest a stratified treatment approach, advocating biologics in patients with poor prognostic markers like ACPA that are failing to attain remission or low disease activity with an initial treatment strategy of synthetic DMARDs [10]. There are, however, insufficient data on prognostic factors in randomized controlled trials (RCTs) of combination DMARDs and biologics to know which approach is best.

We used data from an RCT of combination DMARDs and corticosteroids in early RA - the Combination AntiRheumatic Drugs in Early RA (CARDERA) trial [11] - to examine whether responses to intensive combination treatments differ by ACPA status. Our primary aim was to examine if combination DMARDs and corticosteroids had different effects on radiological progression in ACPApositive and ACPA-negative RA. Our secondary aims were to evaluate if any differential effects also extended to disease activity, disability and quality of life (QoL).

\section{Methods}

\section{Ethical approval}

The CARDERA trial was approved by the South Thames Multicentre Research Ethics Committee (REC Reference: MREC (1) 99/04). Further ethical approval was obtained to process the archived serum for ACPA status from the East of England - Essex Research Ethics Committee (REC Reference: 11/EE/0544). Informed consent was obtained from all patients recruited to the CARDERA trial.

\section{Subjects}

The CARDERA trial recruited patients with early active RA (of less than two years duration) from 42 UK centers; its details have previously been reported $[11,12]$. Patients were randomized to one of four treatment arms: (1) monotherapy with methotrexate; (2) double therapy with methotrexate and ciclosporin; (3) double therapy with methotrexate and prednisolone; (4) triple therapy with methotrexate, ciclosporin and prednisolone. A factorial-design was adopted to allow the simultaneous evaluation of prednisolone and ciclosporin in a $2 \times 2$ design. Treatment groups were well matched with similar baseline characteristics [11]. Patients were assessed every 6 months (for 24 months). Missing data were imputed through last observations carried forward (undertaken in $19 \%$ of patients at 24 months). We restricted our current analysis to the 431 individuals (from 467 recruited) who had their sera archived at baseline and evaluable for ACPA.

\section{Serological assessments}

ACPA-status was evaluated using the commercial ELISA assay, the Axis-Shield DIASTAT anti-CCP2 test (AxisShield, Dundee, UK). All samples were processed in duplicate with a cut-off of $>5$ units $/ \mathrm{ml}$ taken as positive in keeping with the manufacturer's instructions. Rheumatoid factor (RF) had been processed at recruiting center laboratories during the original trial.

\section{Study treatments}

Study treatments comprised: (1) methotrexate (starting at $7.5 \mathrm{mg} /$ week and increasing by $2.5 \mathrm{mg}$ every two weeks to a final dose of $15 \mathrm{mg} /$ week); (2) "step-down" prednisolone (based on the trial by Boers et al. [13], comprising $60 \mathrm{mg} /$ day in week 1 , tapering to $7.5 \mathrm{mg} /$ day in weeks 7 to 28 and thereafter further reduced and stopped by week 36) started with methotrexate; (3) ciclosporin (based on the trial by Pasero et al. [14], comprising $1.5 \mathrm{mg} / \mathrm{kg}$ daily initially, increased gradually to a target dose of $3 \mathrm{mg} / \mathrm{kg}$ daily) started three months after methotrexate. Prednisolone and ciclosporin were given as active tablets or placebos. Intra-articular glucocorticoids (40 mg methylprednisolone with lignocaine) were given (on no more than six occasions) as required. Intramuscular glucocorticoids were allowed but only three doses of $120 \mathrm{mg}$ of depot methylprednisolone could be given in a year.

\section{Outcomes}

The following disease outcomes were assessed: (1) radiological damage - the onset of new erosions at 24 months and modified Larsen scores; (2) disease activity - disease activity scores on a 28-joint count (DAS28); (3) disabilityHealth Assessment Questionnaire (HAQ); (4) QoL- SF-36 


\section{ACPA-POSITIVE PATIENTS}
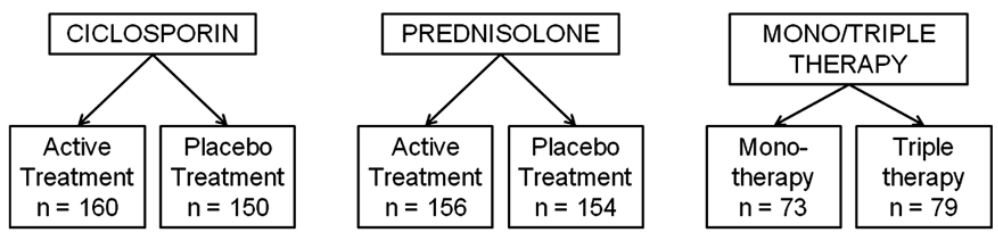

ACPA-NEGATIVE PATIENTS
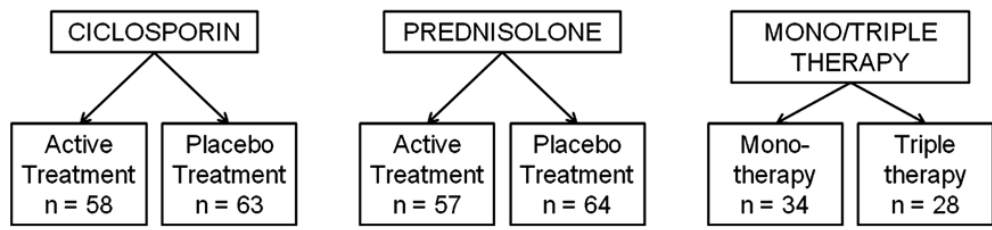

Figure 1 Number of individuals in each treatment group stratified by ACPA status. ACPA, anti-citrullinated protein antibody.

physical (PCS) and mental (MCS) component summary scores and EuroQol.

\section{Statistical analysis}

Baseline differences between ACPA-positive and ACPAnegative patients were evaluated using t-tests, Wilcoxon signed-rank tests or chi-squared tests depending on data type and distribution.

To establish whether treatment response differed by ACPA status, we used a two-staged approach; this minimized the potential for inflation of type I error associated with multiple testing. The first stage used a mixed-effects repeated measures ANOVA model to examine the effect of ACPA, treatment (coded categorically as one of the four randomized treatment arms) and time (assessment visit) on mean changes in each RA outcome (Larsen, DAS28, HAQ, EuroQol, PCS and MCS scores). The key component of this model was an ACPA*treatment interaction term, which established whether treatment responses differed by ACPA status.

The second stage was restricted to outcomes associated with significant $\mathrm{ACPA}^{*}$ treatment interactions and compared mean changes in these outcomes by treatment group at each time point using t-tests in ACPA-positive and ACPA-negative patients. Where the ratio between variances significantly differed from 1 , Satterthwaite's approximation was used to calculate the degrees of freedom for the critical t-statistic. This second stage allowed us to establish which treatments differed in their effects by ACPA status and how these differences changed over time. This analysis followed the original factorial grouping by comparing the following treatment groups (Figure 1): (a) active ciclosporin vs. placebo ciclosporin; (b) active prednisolone vs. placebo prednisolone; (c) triple therapy (methotrexate, cicosporin and prednisolone) vs. monotherapy (methotrexate).

Table 1 Baseline characteristics by ACPA status

\begin{tabular}{llll}
\hline Characteristic & ACPA-positive $(\boldsymbol{n}=\mathbf{3 1 0})$ & ACPA-negative $(\boldsymbol{n}=\mathbf{1 2 1})$ & Group difference \\
\hline Female (number; \%) & $208(67 \%)$ & $89(74 \%)$ & $P=0.193^{2}$ \\
RF positive (number; \%) & $244(79 \%)$ & $47(39 \%)$ & $P<0.001^{2}$ \\
Age (years) & $54.0(46.0,64.0)$ & $55.0(47.0,62.0)$ & $P=0.661^{1}$ \\
Disease duration (months) & $2.00(0.00,5.00)$ & $1.00(0.00,4.00)$ & $P=0.106^{3}$ \\
Larsen score & $7.50(2.50,21.25)^{*}$ & $4.50(1.00,9.50)$ & $P<0.001^{3}$ \\
DAS28 & $5.72(4.91,6.73)$ & $5.96(4.92,6.85)$ & $P=0.305^{1}$ \\
HAQ & $1.62(1.00,2.12)$ & $1.62(1.12,2.12)$ & $P=0.595^{3}$ \\
EuroQol & $0.60(0.15,0.68)$ & $0.58(0.08,0.68)$ & $P=0.552^{3}$ \\
SF-36 PCS & $28.68(23.24,35.95)$ & $28.70(22.90,35.56)$ & $P=0.713^{3}$ \\
SF-36 MCS & $38.64(27.65,53.71)$ & $35.87(25.37,52.95)$ & $P=0.217^{3}$ \\
\hline
\end{tabular}

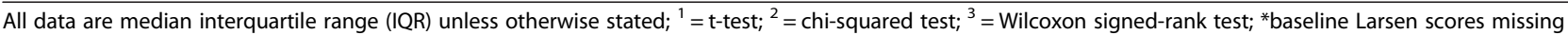
in two ACPA-positive patients. ACPA, anti-citrullinated protein antibody; DAS28, disease activity scores on a 28-joint count; $\mathrm{HAQ}$, Health Assessment Questionnaire; MCS, mental component summary; PCS, physical component summary; RF, rheumatoid factor. 
Table 2 ANOVA results for the effect of ACPA, treatment and time on changes in RA outcomes

\begin{tabular}{|c|c|c|c|c|c|c|c|c|c|c|c|c|}
\hline \multirow[b]{2}{*}{ Effects } & \multicolumn{2}{|c|}{ Larsen } & \multicolumn{2}{|c|}{ DAS28 } & \multicolumn{2}{|c|}{ HAQ } & \multicolumn{2}{|c|}{ EuroQol } & \multicolumn{2}{|c|}{ PCS } & \multicolumn{2}{|c|}{ MCS } \\
\hline & $\mathbf{F}$ & $P$ & $\mathrm{~F}$ & $P$ & $F$ & $P$ & $\mathrm{~F}$ & $P$ & $\mathrm{~F}$ & $P$ & $\mathbf{F}$ & $P$ \\
\hline ACPA & 31.90 & $<0.001$ & 4.02 & 0.045 & 7.25 & 0.007 & 9.72 & 0.002 & 4.07 & 0.044 & 2.29 & 0.131 \\
\hline Time & 16.83 & $<0.001$ & 1.26 & 0.288 & 1.37 & 0.251 & 0.70 & 0.550 & 0.80 & 0.493 & 0.65 & 0.584 \\
\hline Treatment & 9.93 & $<0.001$ & 1.71 & 0.163 & 17.76 & $<0.001$ & 11.47 & $<0.001$ & 5.67 & 0.001 & 1.92 & 0.124 \\
\hline ACPA*Treatment & 7.05 & $<0.001$ & 3.99 & 0.008 & 0.48 & 0.696 & 2.94 & 0.032 & 3.22 & 0.022 & 1.84 & 0.138 \\
\hline
\end{tabular}

$\mathrm{F}=\mathrm{F}$-statistic; $P=P$-value; $\mathrm{ACPA} *$ Treatment $=\mathrm{ACPA} *$ Treatment interaction term. ACPA, anti-citrullinated protein antibody; DAS28, disease activity scores on a 28-joint count; HAQ, Health Assessment Questionnaire; MCS, mental component summary; PCS, physical component summary; RA, rheumatoid arthritis.

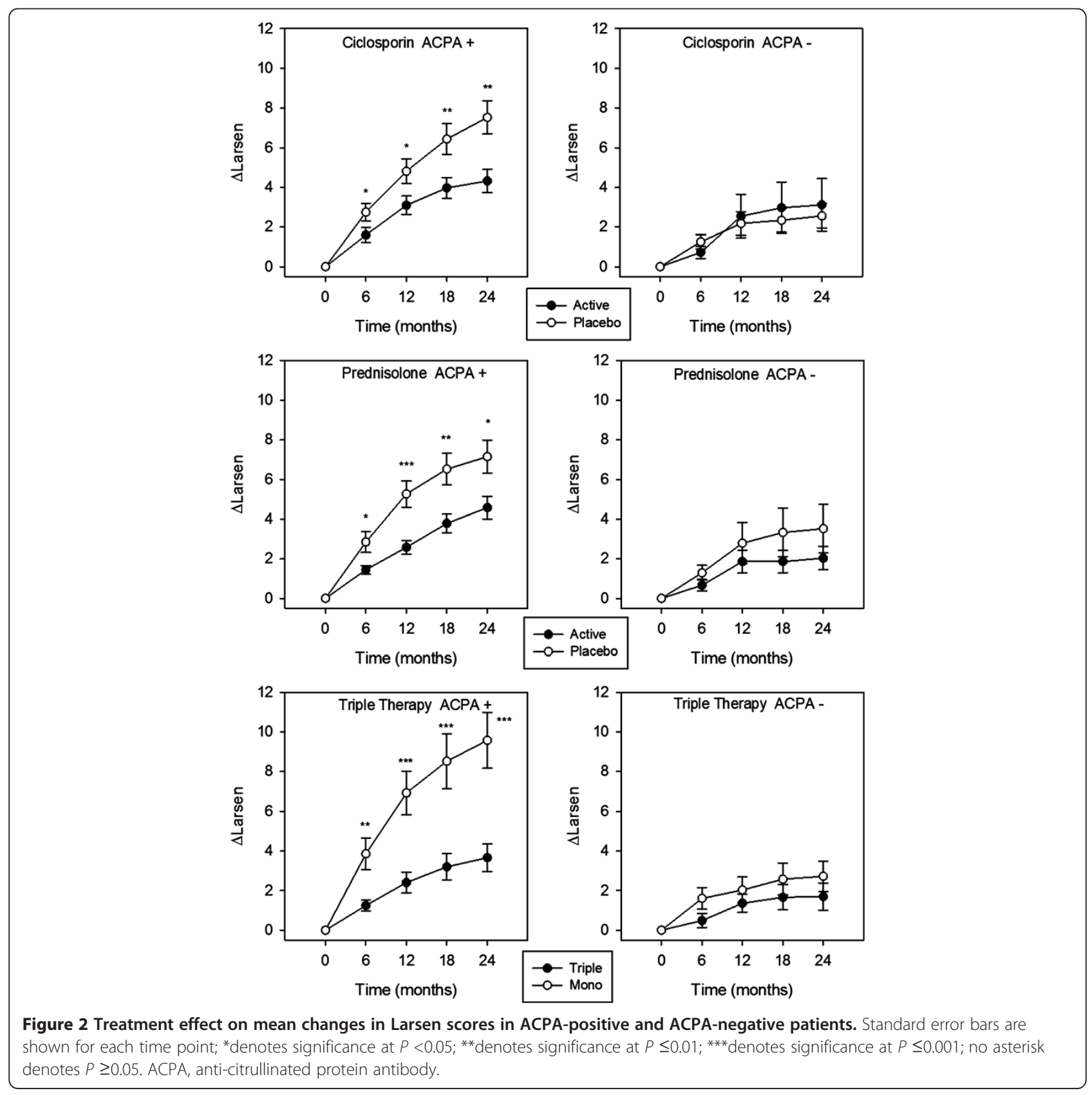


Table 3 Treatment effects on mean changes in Larsen and DAS28 scores in ACPA-positive and ACPA-negative RA

\begin{tabular}{|c|c|c|c|c|c|c|c|c|c|c|}
\hline & \multirow[t]{2}{*}{ Time } & \multicolumn{3}{|l|}{ Ciclosporin } & \multicolumn{3}{|l|}{ Prednisolone } & \multicolumn{3}{|c|}{ Triple vs. monotherapy } \\
\hline & & Ciclosporin & Placebo & $P$ & Prednisolone & Placebo & $P$ & Triple & Mono & $P$ \\
\hline \multicolumn{11}{|l|}{ Larsen } \\
\hline \multirow[t]{4}{*}{$\mathrm{ACPA}+$} & 6 & $1.60(0.37)$ & $2.74(0.44)$ & 0.047 & $1.44(0.22)$ & $2.85(0.53)$ & 0.015 & $1.25(0.28)$ & $3.86(0.80)$ & 0.003 \\
\hline & 12 & $3.09(0.47)$ & $4.81(0.61)$ & 0.027 & $2.58(0.35)$ & $5.26(0.67)$ & 0.001 & $2.41(0.52)$ & $6.92(1.10)$ & $<0.001$ \\
\hline & 18 & $3.97(0.53)$ & $6.43(0.78)$ & 0.010 & $3.79(0.48)$ & $6.52(0.80)$ & 0.004 & $3.20(0.66)$ & $8.52(1.39)$ & 0.001 \\
\hline & 24 & $4.32(0.58)$ & $7.53(0.84)$ & 0.002 & $4.57(0.57)$ & $7.15(0.84)$ & 0.012 & $3.66(0.70)$ & $9.58(1.41)$ & $<0.001$ \\
\hline \multirow[t]{4}{*}{ ACPA- } & 6 & $0.72(0.30)$ & $1.25(0.36)$ & 0.270 & $0.67(0.29)$ & $1.30(0.37)$ & 0.182 & $0.48(0.35)$ & $1.60(0.55)$ & 0.093 \\
\hline & 12 & $2.54(1.11)$ & $2.17(0.59)$ & 0.770 & $1.86(0.58)$ & $2.79(1.04)$ & 0.435 & $1.36(0.47)$ & $2.03(0.66)$ & 0.409 \\
\hline & 18 & $2.97(1.30)$ & $2.33(0.60)$ & 0.660 & $1.86(0.56)$ & $3.33(1.21)$ & 0.275 & $1.66(0.64)$ & $2.57(0.79)$ & 0.388 \\
\hline & 24 & $3.11(1.32)$ & $2.56(0.61)$ & 0.704 & $2.04(0.60)$ & $3.52(1.22)$ & 0.277 & $1.70(0.69)$ & $2.72(0.77)$ & 0.335 \\
\hline \multicolumn{11}{|l|}{ DAS28 } \\
\hline \multirow[t]{4}{*}{$\mathrm{ACPA}+$} & 6 & $-1.61(0.12)$ & $-1.49(0.13)$ & 0.488 & $-1.97(0.12)$ & $-1.13(0.11)$ & $<0.001$ & $-1.98(0.18)$ & $-0.99(0.17)$ & $<0.001$ \\
\hline & 12 & $-1.46(0.12)$ & $-1.19(0.14)$ & 0.147 & $-1.36(0.13)$ & $-1.29(0.13)$ & 0.716 & $-1.48(0.18)$ & $-1.14(0.19)$ & 0.190 \\
\hline & 18 & $-1.49(0.13)$ & $-1.36(0.14)$ & 0.498 & $-1.50(0.14)$ & $-1.36(0.13)$ & 0.479 & $-1.64(0.20)$ & $-1.37(0.20)$ & 0.356 \\
\hline & 24 & $-1.62(0.13)$ & $-1.38(0.15)$ & 0.211 & $-1.62(0.14)$ & $-1.38(0.14)$ & 0.203 & $-1.84(0.19)$ & $-1.36(0.22)$ & 0.087 \\
\hline \multirow[t]{4}{*}{ ACPA- } & 6 & $-1.02(0.21)$ & $-1.42(0.20)$ & 0.173 & $-1.47(0.24)$ & $-1.00(0.17)$ & 0.111 & $-1.43(0.33)$ & $-1.32(0.22)$ & 0.792 \\
\hline & 12 & $-0.94(0.21)$ & $-1.32(0.21)$ & 0.209 & $-1.01(0.21)$ & $-1.25(0.21)$ & 0.421 & $-0.73(0.33)$ & $-1.36(0.33)$ & 0.186 \\
\hline & 18 & $-1.33(0.24)$ & $-1.52(0.20)$ & 0.552 & $-1.33(0.23)$ & $-1.51(0.22)$ & 0.579 & $-1.27(0.37)$ & $-1.62(0.30)$ & 0.462 \\
\hline & 24 & $-1.16(0.22)$ & $-1.49(0.20)$ & 0.256 & $-1.32(0.20)$ & $-1.34(0.21)$ & 0.960 & $-1.27(0.32)$ & $-1.59(0.30)$ & 0.464 \\
\hline
\end{tabular}

Data are mean changes (SE) unless otherwise stated; $P=P$-values from t-tests; Triple = triple DMARD therapy; Mono = monotherapy with methotrexate; time is in months. ACPA, anti-citrullinated protein antibody; DAS28, disease activity scores on a 28 -joint count.

In addition, we calculated odds ratios (ORs) for the development of new erosions with each treatment using binary logistic regression stratified by ACPA status.

$P$-values of $<0.05$ were considered significant. Analyses were performed using SAS version 9.3 (SAS Institute, Cary, NC, USA).

\section{Results}

\section{Patient characteristics}

Of the 431 RA cases, 310 (72\%) were ACPA-positive and 121 (28\%) were ACPA-negative. Baseline characteristics were similar between ACPA subsets with the exception of Larsen scores and RF status (Table 1). ACPA-positive patients had more radiological damage at baseline; the difference in median Larsen scores between ACPA-subsets was 3.00 units $(P<0.001)$. Significantly more ACPA-positive patients were RF-positive $(P<0.001)$. Both ACPA-positive and ACPA-negative patients had median ages in the fifth decade, were mainly female, had severely active RA (median DAS28 scores $>5.1$ ) of a short duration and moderate disability (median HAQ scores 1.62). QoL was moderately impaired (median EuroQol scores 0.58 to 0.60 ).

\section{Radiological progression}

The first analytical step, using the ANOVA model (Table 2), showed that treatment responses differed serologically with a significant ACPA"treatment interactive effect on changes in Larsen scores observed $(P<0.001)$.

The second analytical step, using the factorial approach, showed significant reductions in Larsen score progression in ACPA-positive patients receiving prednisolone, ciclosporin or triple therapy (Figure 2; Table 3). The magnitude of effect was similar with prednisolone and ciclosporin. Those receiving triple therapy had the largest reduction in radiological progression; mean Larsen score increases over 24 months were 3.66 (95\% confidence interval (CI) 2.27 to 5.05) with triple therapy and 9.58 (95\% CI 6.76 to 12.39 ) with monotherapy.

There were no significant treatment effects with any strategy in ACPA-negative patients. These individuals showed substantially less radiological progression (Figure 2; Table 3). The mean Larsen score increase in ACPA-negative patients treated with methotrexate monotherapy over 24 months was 2.72 (95\% CI 1.15 to 4.29); for those receiving triple therapy the mean increase was 1.70 (95\% CI 0.29 to 3.10 ).

Differences in radiological progression between ACPAsubsets were also seen in the proportion of patients developing new erosions (24\% of ACPA-positive patients; $7 \%$ of ACPA-negative patients). Reductions in erosion development in ACPA-positive patients were similar with ciclosporin (OR $0.55 ; 95 \%$ CI 0.31 to $0.96 ; P=0.032$ ) and prednisolone (OR 0.56 ; $95 \%$ CI 0.32 to $0.99 ; P=0.045$ ) when compared with placebo. Triple therapy had the greatest 
impact on reducing new erosions when compared with monotherapy (OR 0.32; 95\% CI 0.14 to $0.72 ; P=0.003$ ). Treatment had no significant impact on preventing erosions in ACPA-negative patients. The ORs for reduction in erosion development in ACPA-negative patients comprised 0.86 (95\% CI 0.16 to $4.23 ; P=1.00)$ and 0.89 (95\% CI 0.17 to $4.38 ; P=1.00)$ with ciclosporin and prednisolone, respectively, compared to placebo and 0.79 (95\% CI 0.06 to 7.53; $P=1.00)$ for triple therapy compared with monotherapy.

\section{Disease activity}

The ANOVA model (Table 2) showed a significant ACPA*treatment interactive effect on changes in DAS28 scores
$(P=0.008)$. Subsequent factorial analysis by treatment showed that prednisolone $(P<0.001)$ and triple therapy $(P<0.001)$ significantly reduced DAS28 scores at six months in ACPA-positive patients (Figure 3; Table 3). No treatment effects were seen at subsequent time points. There were no significant treatment effects in ACPA-negative patients.

\section{Disability}

The ANOVA model (Table 2) showed that although ACPA status $(P=0.007)$ and treatment $(P<0.001)$ influenced changes in HAQ scores no ACPA*treatment interaction
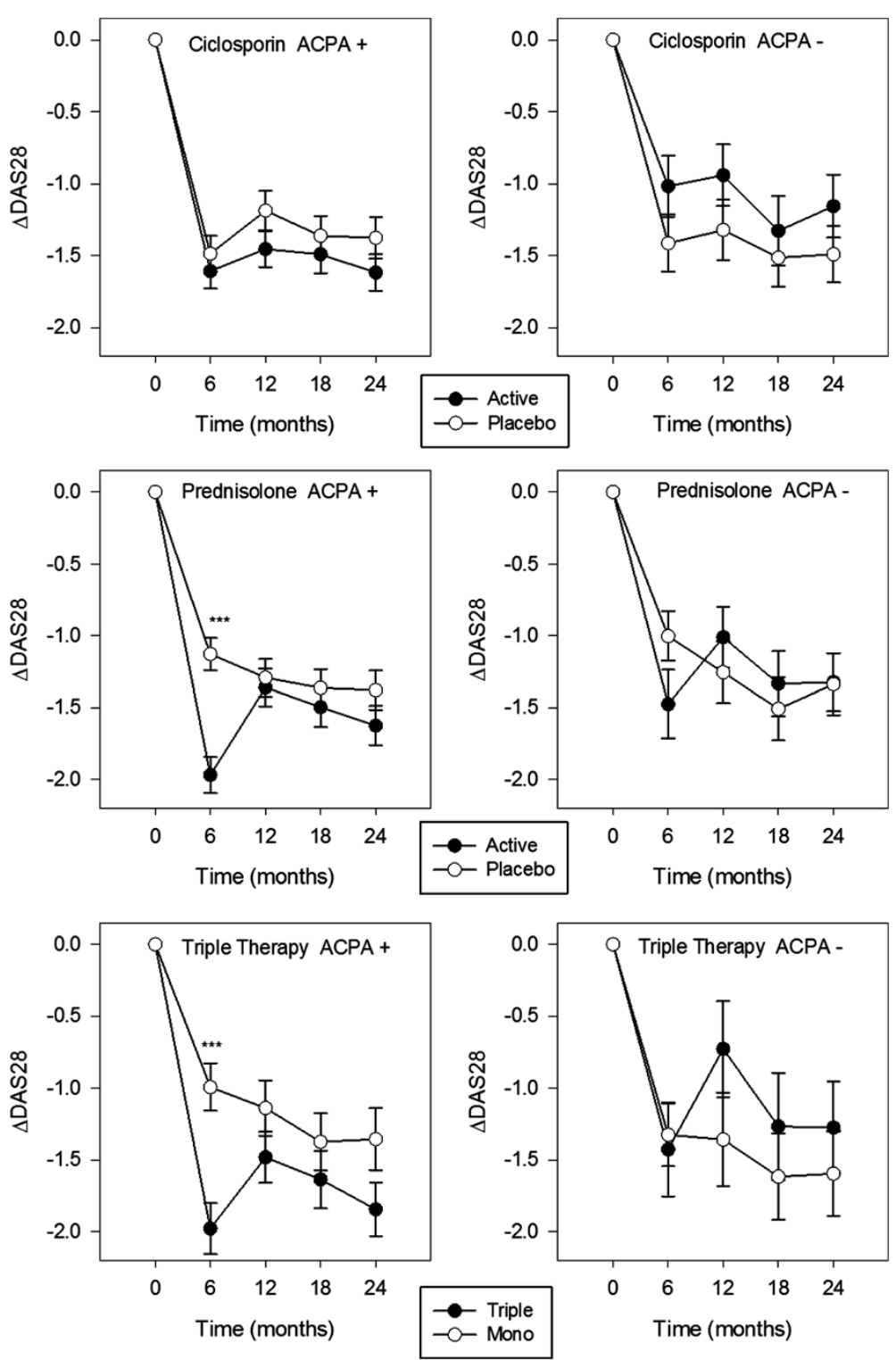

Figure 3 Treatment effect on mean changes in DAS28 scores in ACPA-positive and ACPA-negative patients. Standard error bars are shown for each time point; ${ }^{*}$ denotes significance at $P<0.05$; ${ }^{* *}$ denotes significance at $P \leq 0.01$; ${ }^{* * *}$ denotes significance at $P \leq 0.001$; no asterisk denotes $P \geq 0.05$. ACPA, anti-citrullinated protein antibody; DAS28, disease activity scores on a 28 joint count. 
existed $(P=0.696)$. A factorial analysis was therefore not undertaken.

\section{Quality of life \\ EuroQol}

The ANOVA model (Table 2) showed a significant ACPA*treatment interactive effect on changes in EuroQol scores $(P=0.032)$. Subsequent factorial analysis (Figure 4; Table 4) showed significant improvements in EuroQol scores at 6 months in ACPA-positive patients receiving prednisolone $(P=0.001)$ or triple therapy $(P=0.029)$; a significant effect was also seen at 12 months with ciclosporin $(P=0.044)$. A significant effect of prednisolone on EuroQol scores was seen at all time-points in ACPA-negative patients.

\section{SF-36 PCS}

The ANOVA model (Table 2) showed a significant ACPA*treatment interactive effect on changes in PCS scores $(P=$ 0.022). Factorial analysis (Figure 5; Table 4) showed that in ACPA-positive patients, prednisolone and triple therapy significantly improved PCS scores at 6 and 12 months; ciclosporin also improved PCS scores at 6 months $(P=$ 0.031). In ACPA-negative patients no significant treatment effect on PCS scores was observed.
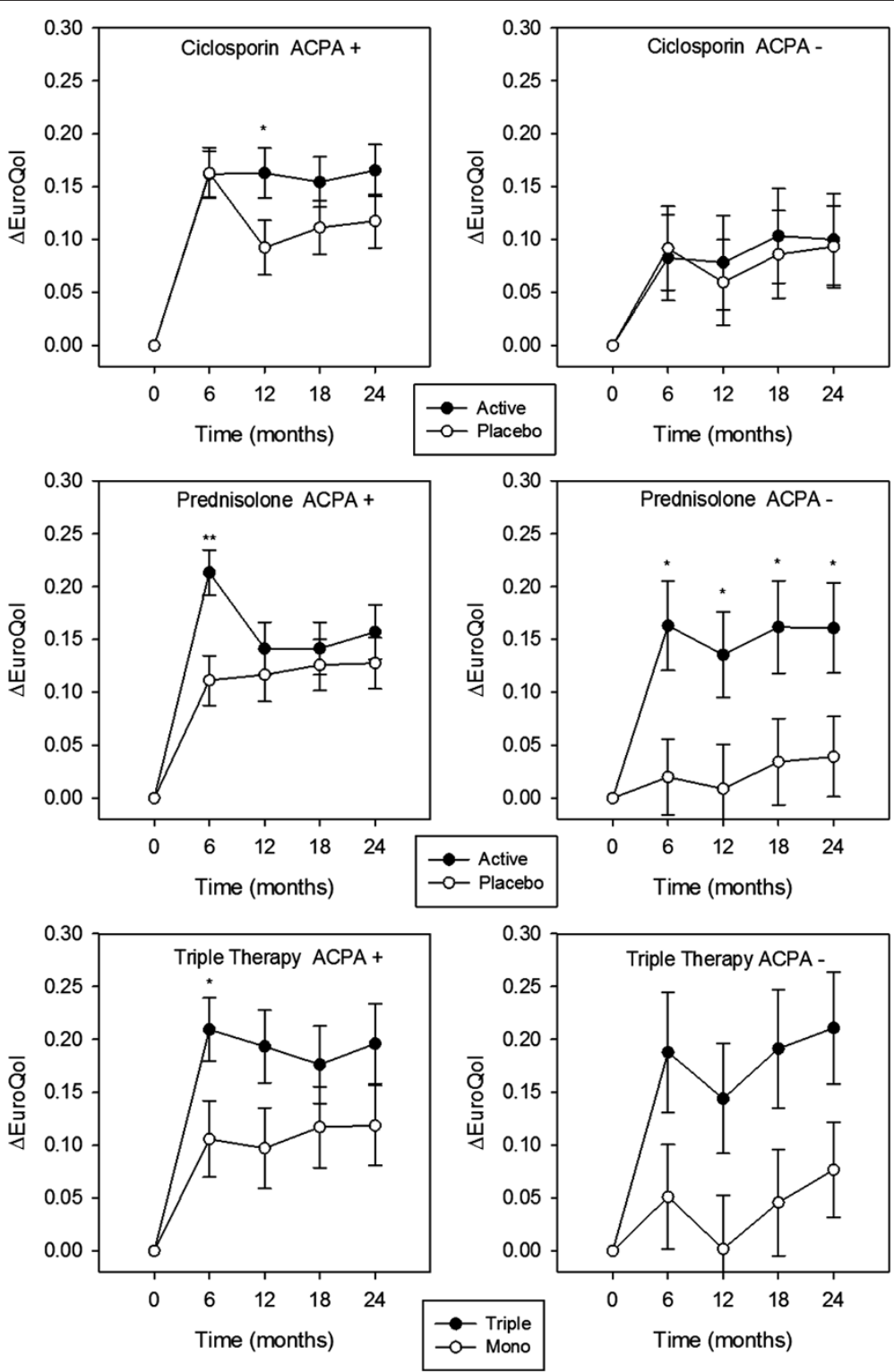

Figure 4 Treatment effect on mean changes in EuroQol scores in ACPA-positive and ACPA-negative patients. Standard error bars are shown for each time-point; ${ }^{*}$ denotes significance at $P<0.05$; ${ }^{* *}$ denotes significance at $P \leq 0.01$; ${ }^{* * *}$ denotes significance at $P \leq 0.001$; no asterisk denotes $P \geq 0.05$. ACPA, anti-citrullinated protein antibody. 
Table 4 Treatment effects on mean changes in EuroQol and PCS scores in ACPA-positive and ACPA-negative RA

\begin{tabular}{|c|c|c|c|c|c|c|c|c|c|c|}
\hline & \multirow[b]{2}{*}{ Time } & \multicolumn{3}{|l|}{ Ciclosporin } & \multicolumn{3}{|l|}{ Prednisolone } & \multicolumn{3}{|c|}{ Triple vs. monotherapy } \\
\hline & & Ciclosporin & Placebo & $P$ & Prednisolone & Placebo & $P$ & Triple & Mono & $P$ \\
\hline \multicolumn{11}{|l|}{ EuroQol } \\
\hline \multirow[t]{4}{*}{$\mathrm{ACPA}+$} & 6 & $0.16(0.02)$ & $0.16(0.02)$ & 0.978 & $0.21(0.02)$ & $0.11(0.02)$ & 0.001 & $0.21(0.03)$ & $0.11(0.04)$ & 0.029 \\
\hline & 12 & $0.16(0.02)$ & $0.09(0.03)$ & 0.044 & $0.14(0.02)$ & $0.12(0.02)$ & 0.481 & $0.19(0.03)$ & $0.10(0.04)$ & 0.063 \\
\hline & 18 & $0.15(0.02)$ & $0.11(0.03)$ & 0.215 & $0.14(0.03)$ & $0.13(0.02)$ & 0.651 & $0.18(0.04)$ & $0.12(0.04)$ & 0.267 \\
\hline & 24 & $0.17(0.02)$ & $0.12(0.03)$ & 0.176 & $0.16(0.03)$ & $0.13(0.02)$ & 0.403 & $0.20(0.04)$ & $0.12(0.04)$ & 0.152 \\
\hline \multirow[t]{4}{*}{ ACPA- } & 6 & $0.08(0.04)$ & $0.09(0.04)$ & 0.876 & $0.16(0.04)$ & $0.02(0.04)$ & 0.011 & $0.19(0.06)$ & $0.05(0.05)$ & 0.074 \\
\hline & 12 & $0.08(0.04)$ & $0.06(0.04)$ & 0.756 & $0.14(0.04)$ & $0.01(0.04)$ & 0.033 & $0.14(0.05)$ & $0.00(0.05)$ & 0.057 \\
\hline & 18 & $0.10(0.04)$ & $0.09(0.04)$ & 0.776 & $0.16(0.04)$ & $0.03(0.04)$ & 0.036 & $0.19(0.06)$ & $0.05(0.05)$ & 0.058 \\
\hline & 24 & $0.10(0.04)$ & $0.09(0.04)$ & 0.908 & $0.16(0.04)$ & $0.04(0.04)$ & 0.035 & $0.21(0.05)$ & $0.08(0.05)$ & 0.057 \\
\hline \multicolumn{11}{|l|}{$P C S$} \\
\hline \multirow[t]{4}{*}{$\mathrm{ACPA}+$} & 6 & $7.96(0.89)$ & $5.15(0.95)$ & 0.031 & $9.15(0.96)$ & $4.01(0.83)$ & $<0.001$ & $10.42(1.30)$ & $2.29(1.16)$ & $<0.001$ \\
\hline & 12 & $5.27(0.84)$ & $4.17(0.94)$ & 0.380 & $6.04(0.93)$ & $3.42(0.84)$ & 0.037 & $7.05(1.23)$ & $3.28(1.27)$ & 0.035 \\
\hline & 18 & $6.11(0.89)$ & $3.87(1.02)$ & 0.097 & $5.51(1.01)$ & $4.53(0.89)$ & 0.472 & 7.77 (1.39) & $4.58(1.44)$ & 0.114 \\
\hline & 24 & $5.77(0.96)$ & $4.91(1.03)$ & 0.544 & $6.36(1.03)$ & $4.34(0.95)$ & 0.150 & $8.33(1.46)$ & $5.53(1.49)$ & 0.180 \\
\hline \multirow[t]{4}{*}{ ACPA- } & 6 & $2.64(1.41)$ & $4.04(1.21)$ & 0.449 & $4.14(1.31)$ & $2.68(1.29)$ & 0.428 & $4.86(2.11)$ & $4.54(1.78)$ & 0.908 \\
\hline & 12 & $3.27(1.35)$ & $4.93(1.15)$ & 0.349 & $3.70(1.25)$ & $4.52(1.25)$ & 0.646 & $3.25(2.07)$ & $5.61(1.76)$ & 0.386 \\
\hline & 18 & $3.86(1.38)$ & $5.06(1.24)$ & 0.516 & $4.41(1.40)$ & $4.55(1.23)$ & 0.937 & $4.72(2.11)$ & $5.88(1.67)$ & 0.664 \\
\hline & 24 & 4.65 (1.69) & $5.17(1.07)$ & 0.793 & $5.51(1.58)$ & $4.40(1.21)$ & 0.575 & 7.44 (2.70) & $6.48(1.36)$ & 0.753 \\
\hline
\end{tabular}

Data are mean changes (SE) unless otherwise stated; $P=P$-values from t-tests; Triple = triple DMARD therapy; Mono = monotherapy with methotrexate; time is in months. ACPA, anti-citrullinated protein antibody; PCS, physical component summary.

\section{SF-36 MCS}

The ANOVA model (Table 2) showed no significant associations between ACPA, time or treatment and MCS scores; no ACPA*treatment interaction was observed $(P=$ 0.138). Factorial analysis was therefore not undertaken.

\section{Discussion}

Our main finding is that combination DMARDs and high-dose tapering corticosteroids are only required to prevent radiological progression in patients with early active RA in whom ACPA is present. In ACPA-positive patients, methotrexate monotherapy resulted in considerable worsening of radiological damage; the average annual Larsen score increase was 4.8 units and 38\% developed new erosions. This was significantly reduced with combination treatment; in ACPA-positive patients receiving triple therapy the average annual Larsen score increase was 1.8 units and 16\% developed new erosions. In contrast, ACPA-negative patients had minimal radiological progression irrespective of the treatment strategy used; the average annual increases in Larsen scores were below the minimal clinically important difference (MCID) of 2.3 units [15] with all treatments and only $7 \%$ developed new erosions.

Our other finding was that the beneficial effect of highdose corticosteroids on reducing disease activity and improving physical health was also confined to ACPA-positive
RA. Only ACPA-positive patients had significant six-month improvements in DAS28 and PCS scores with double and triple therapy regimens incorporating prednisolone. Our findings are consistent with the IMPROVED study, which also found that high-dose corticosteroids had a significantly larger effect on improving disease activity and remission rates in ACPA-positive, as compared to ACPA-negative, inflammatory arthritis patients [16]. The mechanism underlying this differential steroid response is uncertain. The fact these improvements were not maintained over time in CARDERA is expected and consistent with the original COBRA study [13]. Our results support the use of highdose tapering corticosteroids as a bridging therapy in early RA but suggest this treatment strategy would be best reserved for ACPA-positive patients.

The impact of ACPA status on EuroQol responses to combination DMARDs and corticosteroids was less clear, with similar EuroQol improvements observed in ACPApositive and ACPA-negative patients receiving active prednisolone and triple therapy. Interestingly, ACPA-negative patients receiving placebo prednisolone or methotrexate monotherapy had substantially smaller EuroQol improvements (maximal increase of 0.04 and 0.08 units, respectively) when compared to ACPA-positive patients (maximal increase of 0.13 and 0.12 units, respectively). This suggests that methotrexate monotherapy could be more effective at improving QoL in ACPA-positive disease. 

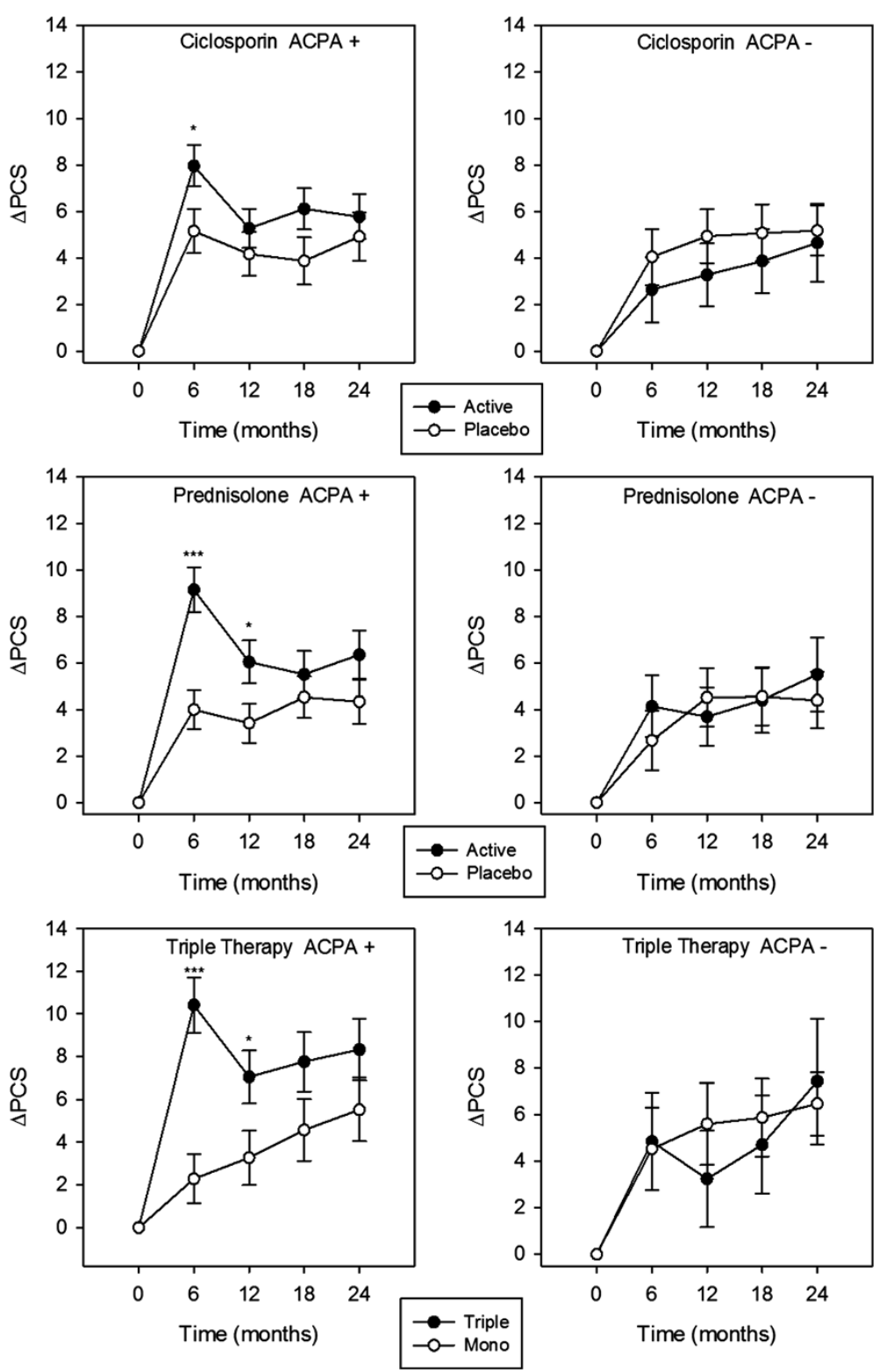

Figure 5 Treatment effect on mean changes in PCS scores in ACPA-positive and ACPA-negative patients. Standard error bars are shown for each time point; * denotes significance at $P<0.05$; ${ }^{* *}$ denotes significance at $P \leq 0.01$; ${ }^{* *}$ denotes significance at $P \leq 0.001$; no asterisk denotes $P \geq 0.05$. ACPA, anti-citrullinated protein antibody; PCS, physical component summary.

To our knowledge, research on the impact of ACPA status on responses to combination DMARDs and corticosteroids is limited to secondary analyses or extension studies of the BeST [17,18] and FIN-RACo [19] trials. Data from the BeST study support our finding that DMARD monotherapy is inadequate at preventing radiological progression in ACPA-positive RA; the presence of ACPA associated with radiological progression in individuals receiving monotherapy (OR for radiological progression: 12.6; 95\% CI 3.0 to 51.9) but not combination therapy with DMARDs and corticosteroids (OR 1.7; 95\% CI 0.5 to 5.4) [17]. This study also reported minimal radiological progression in ACPA-negative patients in all treatment groups. Although the FIN-RACo trial found that combination therapy reduced radiological progression in ACPAnegative, but not ACPA-positive patients, the study had a small sample size, allowed corticosteroids in both treatment arms and had no treatment restrictions for the last three years of follow-up [19]. The impact of ACPA-status on biologic responses has been studied in greater detail, particularly in national registries. Anti-tumor necrosis factor (anti-TNF) therapies appear more effective in ACPAnegative disease [20,21]. Conversely, T-cell and B-cell inhibition with abatacept and rituximab, respectively, appear 
more efficacious in ACPA-positive RA [22,23]. Taken together these findings suggest that treatment responses differ between ACPA-positive and ACPA-negative RA. This highlights a requirement for future RCTs of RA treatments to stratify their analyses by ACPA status.

Our results show that ACPA is an important prognostic biomarker in early RA, with its presence signaling a requirement for intensive combination treatment. The heterogeneous nature of RA alongside the increasing breadth of available therapies means that identifying predictors of treatment responses is a key research goal. Studies have identified several clinical parameters associated with good anti-TNF outcomes; these include not smoking, male gender and a younger age $[20,24]$. Genetic markers also offer promise with a recent large genome-wide association study reporting the first definitive genetic association (in the CD84 gene) with anti-TNF response [25]. Other smaller studies suggest that stimulated whole blood cell pro-inflammatory cytokine levels [26] and serum proteins [27] may be useful in predicting anti-TNF efficacy. These findings are promising but lack clinical utility, since most markers require validation in larger cohorts or associate with only small differences in treatment response. Further work is required to identify predictors of treatment responses in RA.

Our study has a number of strengths. These include its large sample size, the involvement of multiple centers, the measurement of a wide range of outcomes and the use of two-year follow-up data. It also has several limitations. It was a secondary analysis of a published RCT and, therefore, neither its primary hypothesis nor its statistical analysis plan was pre-specified. ACPA status was unknown in $8 \%$ of patients, who were excluded from our analysis. One DMARD, ciclosporin, is not widely used in current practice. Fewer ACPA-negative patients were studied; however, the power to detect a MCID in Larsen scores between combination therapy and monotherapy treatment arms in ACPA-negative patients was higher (86\%) than in ACPApositive patients $(55 \%)$. Finally, the maximal dose of methotrexate was $15 \mathrm{mg} /$ week; higher doses are often used in contemporary clinical care [28].

Different guidelines, constructed using the same evidence base, have drawn alternative conclusions regarding the optimal treatment of early active RA. NICE guidelines recommend offering all patients combination DMARDs and short-term corticosteroids [8]. ACR and EULAR guidelines recommend adopting an individualized approach to treatment intensity based on prognostic factors, such as ACPA $[9,10]$. Our findings favor this latter approach. They show strong evidence that ACPApositive patients benefit from intensive combination therapy but no evidence that combination treatments improve disease outcomes beyond methotrexate monotherapy in ACPA-negative patients. We recommend that future trials in early RA should consider ACPA status when evaluating treatment outcomes. When NICE and other clinical guidelines are updated, the heterogeneity of RA requires consideration, particularly the impact of ACPAstatus on treatment requirements and responses.

\section{Conclusions}

We have demonstrated that the requirement for, and response to, combination DMARDs and high-dose tapering corticosteroids differs between patients with ACPA-positive and ACPA-negative early RA. In our study, intensive combination therapy was only needed to prevent radiological progression in ACPA-positive patients. Additionally, corticosteroids only provided significant improvements in disease activity and physical health outcomes in ACPApositive RA. These findings suggest that ACPA is an important biomarker for guiding treatment decisions in early RA. They support ACR and EULAR RA management guidelines, which recommend an individualized approach to treatment intensity based on prognostic factors such as ACPA.

\section{Abbreviations \\ ACPA: Anti-citrullinated protein antibodies; ACR: American College of Rheumatology; anti-TNF: Anti-tumor necrosis factor; CARDERA: Combination Anti-Rheumatic Drugs in Early RA; Cl: Confidence interval; DAS28: Disease activity score on a 28-joint count; DMARD: Disease-modifying anti-rheumatic drug; EULAR: The European League Against Rheumatism; HAQ: Health assessment questionnaire; MCID: Minimal clinically important difference; MCS: Mental component summary; NICE: National Institute for Health and Care Excellence; OR: Odds ratio; PCS: Physical component summary; QoL: Quality of life; RA: Rheumatoid arthritis; RCT: Randomised controlled trial; RF: Rheumatoid factor.}

\section{Competing interests}

The authors declare that they have no competing interests.

\section{Authors' contributions}

ICS and DLS conceived and designed the study. ICS, MHYM and CD carried out the ACPA ELISA assays. SDS, ICS and CML performed the statistical analysis. SDS, ICS, CML and APC interpreted the data. ICS, SDS, CML, APC and DLS drafted the manuscript. All authors revised the manuscript critically for important intellectual content. All authors read and approved the final manuscript.

\section{Acknowledgements}

This work was supported by Arthritis Research UK (Grant Reference Number 19739 to ICS). It represents research arising from an Arthritis Research UK funded Clinical Research Fellowship (ICS). It also presents independent research funded by the National Institute for Health Research (NIHR) under its Research for Patient Benefit (RfPB) Programme (Grant Reference Number PB-PG-1208-18256). MHYM is a recipient of an NIHR Doctoral Research Fellowship (Grant Reference Number DRF-2009-02-86). The views expressed are those of the authors and not necessarily those of the NHS, the NIHR or the Department of Health. We also acknowledge support from the NIHR Biomedical Research Centre at Guy's and St. Thomas' NHS Foundation Trust in partnership with King's College London. The funders had no role in the study design, data collection and analysis, data interpretation, the writing of the manuscript or the decision to submit the manuscript for publication.

\section{Author details}

'Department of Medical and Molecular Genetics, King's College London, Guy's Hospital, Great Maze Pond, 8th Floor Tower Wing, London SE1 9RT, UK. ${ }^{2}$ Academic Department of Rheumatology, Centre for Molecular and Cellular 
Biology of Inflammation, 1st Floor, New Hunt's House, Guy's Campus, King's College London, Great Maze Pond, London SE1 1UL, UK. ' Department of Rheumatology, 3rd Floor, Weston Education Centre, King's College Hospital, Cutcombe Road, London SE5 9RJ, UK.

Received: 15 September 2013 Accepted: 27 December 2013 Published: 16 January 2014

\section{References}

1. Scott DL, Wolfe F, Huizinga TW: Rheumatoid arthritis. Lancet 2010, 376:1094-1108.

2. van der Helm-van Mil AH, Verpoort KN, Breedveld FC, Toes RE, Huizinga TW: Antibodies to citrullinated proteins and differences in clinical progression of rheumatoid arthritis. Arthritis Res Ther 2005, 7:R949-R958.

3. Eyre S, Bowes J, Diogo D, Lee A, Barton A, Martin P, Zhernakova A, Stahl E, Viatte S, McAllister K, Amos Cl, Padyukov L, Toes RE, Huizinga TW, Wijmenga C, Trynka G, Franke L, Westra HJ, Alfredsson L, Hu X, Sandor C, de Bakker PI, Davila S, Khor CC, Heng KK, Andrews R, Edkins S, Hunt SE, Langford C, Symmons D, et al: High-density genetic mapping identifies new susceptibility loci for rheumatoid arthritis. Nat Genet 2012, 44:1336-1340.

4. Padyukov L, Seielstad M, Ong RT, Ding B, Ronnelid J, Seddighzadeh M, Alfredsson L, Klareskog L: Epidemiological Investigation of Rheumatoid Arthritis study group: a genome-wide association study suggests contrasting associations in ACPA-positive versus ACPA-negative rheumatoid arthritis. Ann Rheum Dis 2011, 70:259-265.

5. Klareskog L, Stolt P, Lundberg K, Kallberg H, Bengtsson C, Grunewald J, Ronnelid J, Harris HE, Ulfgren A-K, Rantapaa-Dahlqvist S, Eklund A, Padyukov L, Alfredsson L: A new model for an etiology of rheumatoid arthritis: smoking may trigger HLA-DR (shared epitope)-restricted immune reactions to autoantigens modified by citrullination. Arthritis Rheum 2006, 54:38-46.

6. Scott IC, Tan R, Stahl D, Steer S, Lewis CM, Cope AP: The protective effect of alcohol on developing rheumatoid arthritis: a systematic review and meta-analysis. Rheumatology 2013, 52:856-867.

7. Daha NA, Toes RE: Rheumatoid arthritis: are ACPA-positive and ACPAnegative RA the same disease? Nat Rev Rheumatol 2011, 7:202-203.

8. Deighton C, O'Mahony R, Tosh J, Turner C, Rudolf M: Guideline Development Group: management of rheumatoid arthritis: summary of NICE guidance. BMJ 2009, 338:b702.

9. Singh JA, Furst DE, Bharat A, Curtis JR, Kavanaugh AF, Kremer JM, Moreland LW, O'Dell J, Winthrop KL, Beukelman T, Bridges SL Jr, Chatham WW, Paulus HE, Suarez-Almazor M, Bombardier C, Dougados M, Khanna D, King CM, Leong AL, Matteson EL, Schousboe JT, Moynihan E, Kolba KS, Jain A, Volkmann ER, Agrawal H, Bae S, Mudano AS, Patkar NM, Saag KG: 2012 update of the 2008 American College of Rheumatology recommendations for the use of disease-modifying antirheumatic drugs and biologic agents in the treatment of rheumatoid arthritis. Arthritis Care Res 2012, 64:625-639.

10. Smolen JS, Landewé R, Breedveld FC, Dougados M, Emery P, Gaujoux-Viala C, Gorter S, Knevel R, Nam J, Schoels M, Aletaha D, Buch M, Gossec L, Huizinga T, Bijlsma JW, Burmester G, Combe B, Cutolo M, Gabay C, Gomez-Reino J, Kouloumas M, Kvien TK, Martin-Mola E, McInnes I, Pavelka K, van Riel P, Scholte M, Scott DL, Sokka T, Valesini G, et al: EULAR recommendations for the management of rheumatoid arthritis with synthetic and biological disease-modifying antirheumatic drugs. Ann Rheum Dis 2010, 69:964-975.

11. Choy EH, Smith CM, Farewell V, Walker D, Hassell A, Chau L, Scott DL, CARDERA (Combination Anti-Rheumatic Drugs in Early Rheumatoid Arhritis) Trial Group: Factorial randomised controlled trial of glucocorticoids and combination disease modifying drugs in early rheumatoid arthritis. Ann Rheum Dis 2008, 67:656-663.

12. Ma MH, Ibrahim F, Walker D, Hassell A, Choy EH, Kiely PD, Williams R, Walsh DA, Young A, Scott DL: Remission in early rheumatoid arthritis: predicting treatment response. J Rheumatol 2012, 39:470-475.

13. Boers $M$, Verhoeven AC, Markusse HM, van de Laar MA, Westhovens $R$, van Denderen JC, van Zeben D, Dijkmans BA, Peeters AJ, Jacobs $P$, van den Brink HR, Schouten HJ, van der Heijde DM, Boonen A, van der Linden S: Randomised comparison of combined step-down prednisolone, methotrexate and sulphasalazine with sulphasalazine alone in early rheumatoid arthritis. Lancet 1997, 350:309-318.

14. Pasero G, Priolo F, Marubini E, Fantini F, Ferraccioli G, Magaro M, Marcolongo R, Oriente P, Pipitone V, Portioli I, Tirri G, Trotta F, Della Casa-Alberighi O: Slow progression of joint damage in early rheumatoid arthritis treated with cyclosporin A. Arthritis Rheum 1996, 39:1006-1015.
15. Bruynesteyn $K$, van der Heijde $D$, Boers $M$, Saudan A, Peloso P, Paulus $H$, Houben H, Griffiths B, Edmonds J, Bresnihan B, Boonen A, van der Linden S: Determination of the minimal clinically important difference in rheumatoid arthritis joint damage of the Sharp/van der Heijde and Larsen/Scott scoring methods by clinical experts and comparison with the smallest detectable difference. Arthritis Rheum 2002, 46:913-920.

16. Wevers-de Boer K, Visser K, Heimans L, Ronday HK, Molenaar E, Groenendael JH, Peeters AJ, Westedt M-L, Collée G, de Sonnaville PBJ, Grillet BA, Huizinga TW, Allaart CF: Remission induction therapy with methotrexate and prednisone in patients with early rheumatoid and undifferentiated arthritis (the IMPROVED study). Ann Rheum Dis 2012, 71:1472-1477.

17. de Vries-Bouwstra JK, Goekoop-Ruiterman YP, Verpoort KN, Schreuder GM, Ewals JA, Terwiel JP, Ronday HK, Kerstens PJ, Toes RE, de Vries RR, Breedveld FC, Dijkmans BA, Huizinga TW, Allaart CF: Progression of joint damage in early rheumatoid arthritis: association with HLA-DRB1, rheumatoid factor, and anti-citrullinated protein antibodies in relation to different treatment strategies. Arthritis Rheum 2008, 58:1293-1298.

18. van den Broek M, Dirven L, Klarenbeek NB, Molenaar TH, Han KH, Kerstens PJ, Huizinga TW, Dijkmans BA, Allaart CF: The association of treatment response and joint damage with ACPA-status in recent-onset RA: a subanalysis of the 8-year follow-up of the BeSt study. Ann Rheum Dis 2012, 71:245-248.

19. Mustila A, Korpela M, Haapala AM, Kautiainen H, Laasonen L, Mottonen T, Leirisalo-Repo M, Ilonen J, Jarvenpaa S, Luukkainen R, Hannonen P: Anti-citrullinated peptide antibodies and the progression of radiographic joint erosions in patients with early rheumatoid arthritis treated with FIN-RACo combination and single disease-modifying antirheumatic drug strategies. Clin Exp Rheumatol 2011, 29:500-505.

20. Canhao H, Rodrigues AM, Mourao AF, Martins F, Santos MJ, Canas-Silva J, Polido-Pereira J, Pereira Silva JA, Costa JA, Araujo D, Silva C, Santos H, Duarte C, da Silva JA, Pimentel-Santos FM, Branco JC, Karlson EW, Fonseca JE, Solomon DH: Comparative effectiveness and predictors of response to tumour necrosis factor inhibitor therapies in rheumatoid arthritis. Rheumatology 2012, 51:2020-2026.

21. Potter $C$, Hyrich KL, Tracey A, Lunt M, Plant D, Symmons DP, Thomson W, Worthington J, Emery P, Morgan AW, Wilson AG, Isaacs J, Barton A, BRAGGSS: Association of rheumatoid factor and anti-cyclic citrullinated peptide positivity, but not carriage of shared epitope or PTPN22 susceptibility variants, with anti-tumour necrosis factor response in rheumatoid arthritis. Ann Rheum Dis 2009, 68:69-74. Erratum in: Ann Rheum Dis 2011, 70:1519.

22. Gottenberg JE, Ravaud P, Cantagrel A, Combe B, Flipo RM, Schaeverbeke T, Houvenagel E, Gaudin P, Loeuille D, Rist S, Dougados M, Sibilia J, Le Loet X, Marcelli C, Bardin T, Pane I, Baron G, Mariette X: Positivity for anti-cyclic citrullinated peptide is associated with a better response to abatacept: data from the 'Orencia and Rheumatoid Arthritis'registry. Ann Rheum Dis 2012, 71:1815-1819.

23. Isaacs JD, Cohen SB, Emery P, Tak PP, Wang J, Lei G, Williams S, Lal P, Read SJ: Effect of baseline rheumatoid factor and anticitrullinated peptide antibody serotype on rituximab clinical response: a meta-analysis. Ann Rheum Dis 2013, 72:329-336.

24. Kleinert S, Tony H-P, Krause A, Feuchtenberger M, Wassenberg S, Richter C, Rother E, Spieler W, Gnann H, Wittig BM: Impact of patient and disease characteristics on therapeutic success during adalimumab treatment of patients with rheumatoid arthritis: data from a German noninterventional observational study. Rheumatol Int 2012, 32:2759-2767.

25. Cui J, Stahl EA, Saevarsdottir S, Miceli C, Diogo D, Trynka G, Raj T, Mirkov MU, Canhao H, Ikari K, Terao C, Okada Y, Wedren S, Askling J, Yamanaka H, Momohara S, Taniguchi A, Ohmura K, Matsuda F, Mimori T, Gupta N, Kuchroo M, Morgan AW, Isaacs JD, Wilson AG, Hyrich KL, Herenius M, Doorenspleet ME, Tak P-P, Crusius JB, et al: Genome-wide association study and gene expression analysis identifies CD84 as a predictor of response to etanercept therapy in rheumatoid arthritis. PLoS Genet 2013, 9:e1003394.

26. Kayakabe K, Kuroiwa T, Sakurai N, Ikeuchi H, Kadiombo AT, Sakairi T, Kaneko Y, Maeshima A, Hiromura K, Nojima Y: Interleukin-1 measurement in stimulated whole blood cultures is useful to predict response to anti-TNF therapies in rheumatoid arthritis. Rheumatology 2012, 51:1639-1643

27. Ortea I, Roschitzki B, Ovalles JG, Longo JL, de la Torre I, Gonzalez I, Gomez-Reino JJ, Gonzalez A: Discovery of serum proteomic biomarkers 
for prediction of response to infliximab (a monoclonal anti-TNF antibody) treatment in rheumatoid arthritis: an exploratory analysis. J Proteomics 2012, 77:372-382.

28. Visser K, van der Heijde D: Optimal dosage and route of administration of methotrexate in rheumatoid arthritis: a systematic review of the literature. Ann Rheum Dis 2009, 68:1094-1099.

doi:10.1186/ar4439

Cite this article as: Seegobin et al: ACPA-positive and ACPA-negative rheumatoid arthritis differ in their requirements for combination DMARDs and corticosteroids: secondary analysis of a randomized controlled trial. Arthritis Research \& Therapy 2014 16:R13.

\section{Submit your next manuscript to BioMed Central and take full advantage of:}

- Convenient online submission

- Thorough peer review

- No space constraints or color figure charges

- Immediate publication on acceptance

- Inclusion in PubMed, CAS, Scopus and Google Scholar

- Research which is freely available for redistribution 